Check for updates

Cite this: RSC Adv., 2021, 11, 1841

\title{
Stabilizing decontamination foam using surface- modified silica nanoparticles containing chemical reagent: foam stability, structures, and dispersion properties $\dagger$
}

\author{
In-Ho Yoon, (D) *a Suk Bon Yoon, ${ }^{\text {b }}$ Youngho Sihn, ${ }^{a}$ Man-Soo Choi, ${ }^{a}$ \\ Chong-Hun Jung (D) and Wang-Kyu Choi ${ }^{a}$
}

\begin{abstract}
The stabilization of decontamination foams containing a chemical reagent is a crucial requirement for their use in the decontamination of nuclear power plants. We have investigated the effects on decontamination foam stability of adding silica nanoparticles (NPs) modified with various functional groups, namely propyl $\left(-\mathrm{CH}_{3}\right)$, amine $\left(-\mathrm{NH}_{2}\right)$, and thiol $(-\mathrm{SH})$ groups. The surface properties of these silica NPs were characterized with ATR-FTIR, solid NMR, and TGA analyses. We also established that the agglomeration in such foams of the amine-modified silica NPs is weaker than that of the other modified silica NPs due to their thorough dispersion in the liquid film. Further, the foam containing amine-modified silica NPs was found to be stable for $60 \mathrm{~min}$ at a $\mathrm{pH}$ of 2, i.e. under decontamination conditions. The bubble structure analysis showed that this decontamination foam has a bubble count that is approximately 5-8 times higher than the foams containing NPs modified with the other functional groups, which indicates that the decontamination foam with amine-modified silica NPs has the best foam structure of the three investigated foams. The well-dispersed and smaller amine-modified silica NPs enhance the foam stability by providing a barrier between the gas bubbles and delaying their coalescence. In contrast, the thioland propyl-modified silica NPs form aggregates with large diameters that reduce the maximum capillary pressure of coalescence and hence decrease the foam stability.
\end{abstract}

Received 6th September 2020

Accepted 30th November 2020

DOI: $10.1039 / \mathrm{dOra07644a}$

rsc.li/rsc-advances
LAPONITE ${ }^{8},{ }^{8-10}$ and silica NPs. ${ }^{11-14}$ It is difficult to treat secondary wastes containing organic matter after foam decontamination, so inorganic stabilizers are preferred over organic stabilizers.

In our approach, the foam consists of a two-phase system of surfactants and silica NPs; the NPs act as surface modifiers that increase the stability of dispersions in the two-phase system. ${ }^{15-18}$ However, because the role of NP-surfactant interactions in the interfacial behavior of fluid/fluid systems is not yet well understood, the mechanism of NP-surfactant interactions is the subject of increasing research attention. ${ }^{19}$ The complexity of such interfacial behavior in two-phase surfactant-NP systems is increased by the electrostatic attraction and repulsion of ionic surfactants. In general, unmodified silica NPs, such as hydrophilic silica, are not surface active, but they can strongly influence the interfacial behavior in two-phase surfactant-NP systems. ${ }^{20,21}$

Although a number of valuable studies of the interfacial behavior of two-phase surfactant-NP systems have been conducted, their focus has mainly been negatively charged silica NPs and the cationic surfactant cetyl trimethyl ammonium bromide (CTAB)..$^{20,22-28}$ According to the results of these studies, the electrostatic attraction between the negatively charged
${ }^{a}$ Decommissioning Technology Research Division, Korea Atomic Energy Research Institute, 111, Daedeok-daero 989 Beon-gil, Yuseong-gu, Daejeon, 305-353, Republic of Korea. E-mail: ihyoon@kaeri.re.kr; Tel: +82-42-868-8281

${ }^{b}$ Decommissioning Research Institute Preparation Unit, Korea Hydro \& Nuclear Power Co., Ltd. (KHNP), Gyeongju, 38120, Republic of Korea

$\dagger$ Electronic supplementary information (ESI) available. See DOI: 10.1039/d0ra07644a 
surfaces of the silica NPs and the cationic surfactant (CTAB) enhances surfactant adsorption on the surfaces of the silica NPs. In contrast, anionic surfactants do not interact with the surfaces of negatively charged silica NPs. The interfacial tension of a sodium dodecyl sulfate (SDS) solution below its critical micelle concentration is lower in the presence of negatively charged silica NPs. ${ }^{29-33}$

The change in the hydrophobic and hydrophilic properties that result from surfactant adsorption is the main reason that silica NPs adsorb onto the liquid interface. ${ }^{25,34}$ In this study, the surface-modified silica NPs act as surface-modifying agents that enhance the interfacial properties of the two-phase surfactantNP system and thereby increase its stability. ${ }^{22,23,25,26,35}$ Various surface treatments can be used to adjust the dispersion of the silica NPs and thus the foam stability, particularly the addition of various functional groups that promote electrostatic interactions with their surfaces. ${ }^{36-41}$ Recently, researchers reported the enhancement of foam stability that results from modifying silica NPs with positively charged polyethylenimine ${ }^{36}$ or enhancing their partial hydrophobicity with the addition of $\left(\mathrm{CH}_{3}\right)_{2} \mathrm{SiCl}_{2}{ }^{40}$ However, surface-modified silica NPs have not previously been used as stabilizers in decontamination foams. In this study, we synthesized silica NPs with surfaces that had been modified with various functional groups with the aim of increasing their dispersion in decontamination foams by minimizing the interaction between the silica NPs and the surfactant, and thus enhancing the foam stability. We analyzed the physical properties of the silica NPs, and examined the resulting foam stability and dispersion behavior with the aim of developing a new decontamination foam formulation (Fig. 1). We investigated (i) the characteristics of the surface-modified silica NPs, (ii) the surface charges, size distributions, and dispersion properties of these silica NPs in decontamination foam solutions, and (iii) the foam stability and structures of the
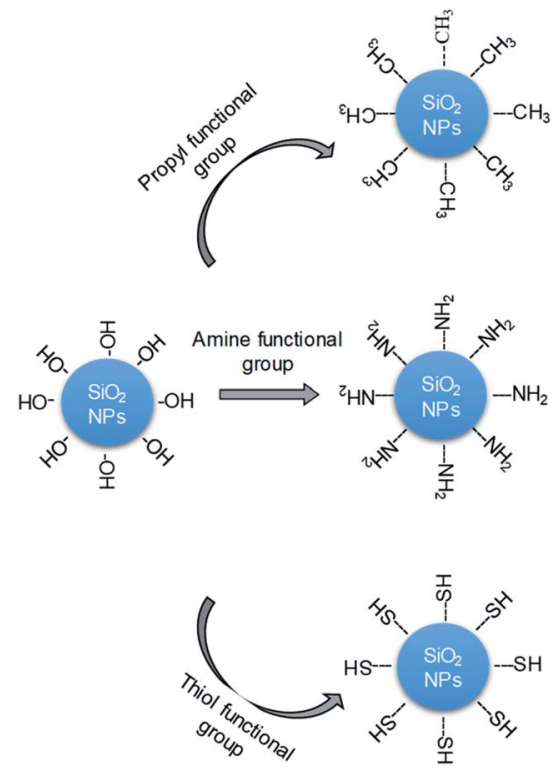

Fig. 1 Schematic diagram of the modification of the surfaces of silica NPs (bare) with propyl $\left(-\mathrm{CH}_{3}\right)$, amine $\left(-\mathrm{NH}_{2}\right)$, or thiol $(-\mathrm{SH})$ groups. decontamination foams containing modified silica NPs. This is the first time surface-modified silica NPs have been used to enhance the stability of decontamination foams. The new formulation with amine-modified silica NPs was found to exhibit enhanced foam stability because of the increase in the dispersibility of the silica NPs.

\section{Experimental}

\section{Synthesis and modification of silica nanoparticles}

Non-porous silica NPs were synthesized by using an established method..$^{42}$ In a typical procedure, silica seed NPs were synthesized by adding tetraethylorthosilicate (TEOS; $25 \mathrm{~mL}$ ) into a mixture consisting of absolute ethanol $(1000 \mathrm{~mL})$, deionized $\mathrm{H}_{2} \mathrm{O}(10 \mathrm{~mL})$, and $28 \mathrm{wt} \% \mathrm{NH}_{4} \mathrm{OH}(25 \mathrm{~mL})$, and then stirring for $24 \mathrm{~h}$ at the ambient temperature. The silica seed solution (24 $\mathrm{mL}$ ) was then suspended in a solution of absolute ethanol (1000 $\mathrm{mL})$, DI $\mathrm{H}_{2} \mathrm{O}(80 \mathrm{~mL})$, and $28 \mathrm{wt} \% \mathrm{NH}_{4} \mathrm{OH}(40 \mathrm{~mL})$. After stirring for $0.5 \mathrm{~h}$, tetraethylorthosilicate $(40 \mathrm{~mL})$ was added into the above reaction mixture and stirring was continued for $6 \mathrm{~h}$ under the same conditions. Finally, tetraethylorthosilicate $(80 \mathrm{~mL})$ was added to the mixture, which was then stirred for $6 \mathrm{~h}$. Various functional groups were added to the silica NPs by reacting the particle surfaces with the silane coupling agents (3-aminopropyl)trimethoxysilane $(+96 \%, 281778$, Aldrich) for amine functionalization $\left(-\mathrm{NH}_{2}\right)$, (3-mercaptopropy)trimethoxysilane $(+95 \%, 175617$, Aldrich) for thiol functionalization (-SH), and $n$ propyltriethoxysilane $(97 \%, 539317$, Aldrich) for propyl functionalization $\left(-\mathrm{CH}_{3}\right)$. In these surface modifications, $1.0 \mathrm{~g}$ synthesized silica NPs in $100 \mathrm{~mL}$ DI water was magnetically stirred with $1 \% \mathrm{~mol}_{\text {silane }} \mathrm{mol}_{\text {silica }}{ }^{-1}$ silane coupling agents. The concentrations of the reacted silane agents were typical of silica surface modifications. The modified silica NPs were separated by centrifugation at $100000 \mathrm{~g}$ for $15 \mathrm{~min}$. The samples were washed with ethanol/DI water and freeze-dried over $24 \mathrm{~h}$.

\section{Preparation of the decontamination foams}

The surfactant Elotant ${ }^{\mathrm{TM}}$ Milcoside 100 (EM 100) (alkyl polyglucoside) for the preparation of the decontamination foam was provided by LG Household \& Health Care, and it contained 10 alkyl chains on average. All solutions were prepared by using deionized (DI) water from a Milli-Q water system. To examine the effects of the silica NPs modified with amine, thiol, and propyl groups on foam stability, $100 \mathrm{~mL}$ of $\mathrm{DI} \mathrm{H}_{2} \mathrm{O}$ was added to a $1.0 \% \mathrm{v} / \mathrm{v}$ solution of EM100 at $\mathrm{pH} 2$ adjusted with $1 \mathrm{M} \mathrm{HNO}_{3}$ in a $100 \mathrm{~mL}$ beaker.

\section{Foam stability and structure analyses}

In all the foam stability and structure experiments, the foam and its liquid height/volume were analyzed by using a Dynamic Foam Analyzer (DFA-100, KR̂SSS, Germany), while the foam structure was simultaneously characterized (particularly the bubble size distribution and the thickness of the liquid layers within the foam). Air was injected through a sintered glass filter at the bottom of a cylindrical glass vessel (40 mm inner diameter) during foaming. The diameter and thickness of the glass 
filter were $4.4 \mathrm{~cm}$ and $0.4 \mathrm{~cm}$ respectively. The pore size of the filter was $16-40 \mu \mathrm{m}$. The initial liquid volume was $0.06 \mathrm{~L}$; the gas flow was $0.2 \mathrm{~L} \mathrm{~min}^{-1}$ during foaming and was stopped after $60 \mathrm{~s}$. The foam height and liquid height were analyzed by using a light-emitting diode panel and a photon detector positioned at the front and back of the column, and were continuously checked by analyzing the light transmission through the glass column..$^{\mathbf{4 3 4}}$ The total foam height was $20 \mathrm{~cm}$ (approximately $0.25 \mathrm{~L}$ ) in all determinations.

A camera with a scanning area of $1.05 \mathrm{~cm} \times 0.75 \mathrm{~cm}$ was positioned $5.5 \mathrm{~cm}$ above the glass filter to determine the foam size distribution. By making use of the principle of total reflection, a prism enables the $2 \mathrm{D}$ structure analysis of foams located along the path of the light. The glass and liquid have similar refractive indices, so the light incident on a foam lamella is partially diffracted and thus transmitted into the bubble. In contrast, glass and air have different refractive indexes, so the light hitting the gas bubbles is completely reflected. The subsequent high-contrast images were investigated by using foam analysis software, and the bubble size distribution was recorded every $2 \mathrm{s.}^{44}$

\section{Analysis}

The synthesized silica NPs and those modified with amine, thiol, and propyl groups were characterized by using various analytical tools. Scanning electron microscopy (SEM) (S-4300, Hitachi, Japan) was used to determine the shapes and sizes of the NPs. The surface areas of the NPs were determined by using the Brunauer-Emmett-Teller (BET) method with a Micromeritics ASAP 2420 (USA) analyzer at $77 \mathrm{~K}$. Pre-heated vacuum degassing was performed on the samples at $423 \mathrm{~K}$ for $2 \mathrm{~h}$ prior to the isotherm measurements. The transmittance spectra of the modified silica NPs were recorded with ATR-FTIR (Alpha-P, Bruker, USA). These measurements were carried out at a resolution of $4 \mathrm{~cm}^{-1}$ with 64 scans over the range $600-4000 \mathrm{~cm}^{-1}$. The extent of functionalization of the silica NPs was investigated with elemental analysis (EA, Flash 2000 Thermo, USA) and thermogravimetric analysis (TGA, TG 209 F1 Libra Netzsch, Germany). In the TGA, the modified NPs were heated from 25 to $800{ }^{\circ} \mathrm{C}$ at a heating rate of $20^{\circ} \mathrm{C} \mathrm{min}^{-1}$ in a $\mathrm{N}_{2}$ atmosphere. The heating temperatures were maintained at 150,350 , and $550{ }^{\circ} \mathrm{C}$ for $30 \mathrm{~min}$ to precisely quantify the surface bound components. We calculated the surface densities of nitrogen $\left(-\mathrm{NH}_{2}\right)$, carbon $\left(-\mathrm{CH}_{3}\right)$, and sulfur $(-\mathrm{SH})$, by using the equation

$$
\begin{aligned}
\text { Surface density }= & \text { element mass per functionalized silica mass } \\
& \times \text { molecular weight of element } \\
& \times \text { Avogadro } \mathrm{s} \text { number } \\
& \div \text { surface area of the silica }
\end{aligned}
$$

The surface area of functional groups was determined to be $15.4 \mathrm{~m}^{2} \mathrm{~g}^{-1}$ by performing BET analyses.

Solid-state proton nuclear magnetic resonance (1H NMR) spectra were recorded with a Bruker AVANCE III HD (Bruker, Germany) at the KBSI Western Seoul Center. The analysis was performed at 9.4 $\mathrm{T}$ by using a HX CPMAS probe with a $4 \mathrm{~mm}$ o.d. zirconia rotor. Tetramethylsilane (TMS) solution was used as the external reference. The sizes and surface charges of the modified NPs were determined by using a DLS device (Malvern Zetasizer nano-ZS90, Malvern, UK).

The dispersion stabilities of the pure silica NPs and the surface-modified silica NPs were analyzed by using a Turbiscan (Turbiscan Lab, Formulaction, France). Sedimentation phenomena were assessed by using an optical analyzer, Turbiscan Lab (Formulaction, France), which uses multiple light scattering to characterize dispersion conditions. The Turbiscan Lab was used to detect and characterize the destabilization of dispersions with various concentrations, i.e. their coalescence, flocculation, creaming, and sedimentation. The viscosity properties of the various foam formulations were analyzed by using a rheometer (Brookfield Eng. \& Lab. Inc., LVDV-III ULTRA) at a shear rate of $60 / \mathrm{s}^{-1}$ for $2 \mathrm{~min}$ (120 points) and Rheocalc 32 software.

\section{Results and discussion}

\section{Characteristics of the surface-modified silica NPs}

We determined the properties of the bare and modified silica NPs by using various analytical tools, and our results clearly show that the desired functional groups were grafted onto the silica surfaces. Attenuated total reflectance Fourier transform infrared (ATR-FTIR) and solid NMR analyses were used to characterize the functional groups grafted onto the surfaces of the silica NPs. Fig. 2 shows the ATR-FTIR spectra of the bare and modified surfaces of the silica NPs. Intense silica peaks are evident for all samples in the range $800-1100 \mathrm{~cm}^{-1}$, particularly the stretching vibrations of $\mathrm{Si}-\mathrm{O}-\mathrm{Si}$ and $\mathrm{Si}-\mathrm{OH}$ at 1050 and $800 \mathrm{~cm}^{-1}$ respectively. Vibration peaks due to the alkyl chains $\mathrm{CH}_{3}$ and $\mathrm{CH}_{2}$ were observed only for the modified silica NPs in the range $2800-3000 \mathrm{~cm}^{-1}$, whereas there are no such peaks for the bare silica NPs (Fig. 2 (inset)). Since the silane coupling agents contain both functional groups and alkyl chain groups, the observed alkyl peaks demonstrate that the silane coupling agents are immobilized on the surfaces of the silica NPs. Bands

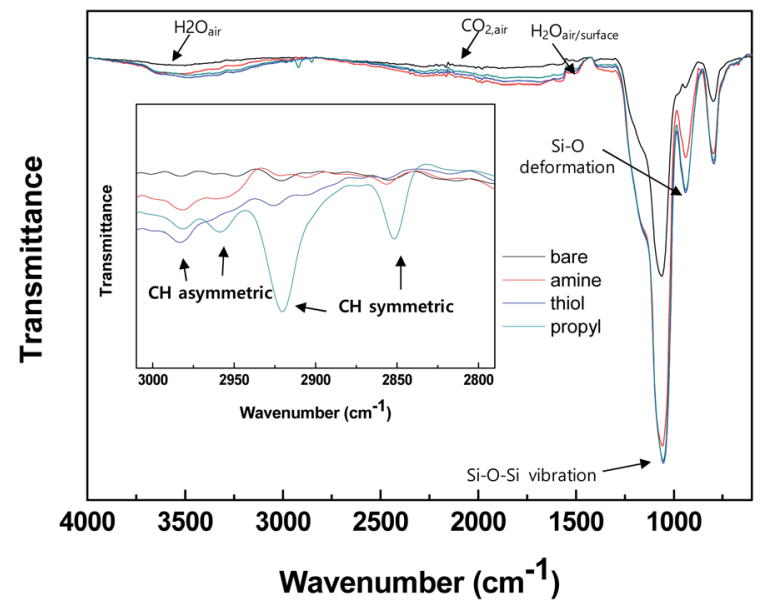

Fig. 2 ATR-FTIR spectra of the silica NPs: bare and functionalized with amine, thiol, or propyl groups. 
due to functional groups are not evident in the spectra for the modified silica NPs probably because of their overlap with the intense silica and $\mathrm{CO}_{2} / \mathrm{H}_{2} \mathrm{O}_{\text {(air) }}$ peaks.

${ }^{1} \mathrm{H}$ NMR analysis was carried out to confirm the presence of grafted functional groups on the surfaces of the silica NPs (Fig. 3). For all the modified silica NP samples, intense signals are evident due to water adsorbed onto the silica NPs and the alkoxy and alkyl chain groups of the grafted silane coupling agents. ${ }^{45-47}$ The spectra of the samples modified with different functional groups contain weak signals at different positions according to the characteristics of the functional groups, which match previously reported values, i.e., $3.5 \mathrm{ppm}\left(-\mathrm{NH}_{2}\right.$ silica), $2.7 \mathrm{ppm}$ (-SH silica), and $0.9 \mathrm{ppm}\left(-\mathrm{CH}_{3}\right.$ silica) ${ }^{48-51}$ These results confirm that the silane coupling agents have been successfully grafted onto the surfaces of the silica NPs.

\section{Elemental and TGA analyses for the silica NPs modified with various functional groups}

The thermolysis profiles shown in Fig. 4 of the modified silica NPs were obtained with TGA to determine the amounts of organic groups on the modified silica NP surfaces. The heating temperatures were maintained at 150,350 , and $550{ }^{\circ} \mathrm{C}$ to perform the complete thermolysis of each component of the modified silica NPs. The profiles contain three abrupt mass loss regions. The first mass loss (4\%) occurs during the period 0$10 \mathrm{~min}$ from 25 to $150{ }^{\circ} \mathrm{C}$ and is due to the loss of surfaceadsorbed water. The next region (approximately 2\%) occurs from 150 to $350{ }^{\circ} \mathrm{C}$ and is probably due to the loss of physically entrapped or adsorbed organics such as ethanol or external impurities. The last region occurs from 350 to $550{ }^{\circ} \mathrm{C}$ and is ascribed to the loss of the silanes grafted onto the silica NP surfaces. The complete decomposition of the silanes occurs above $450{ }^{\circ} \mathrm{C}$ due to the cleavage of the bonds between silicon atoms and hydrocarbon chains. ${ }^{52}$ Thus substantial mass loss (1.81-2.49\%) clearly arises for the modified silica NPs in the range $350-550{ }^{\circ} \mathrm{C}$, which confirms that the silanes containing

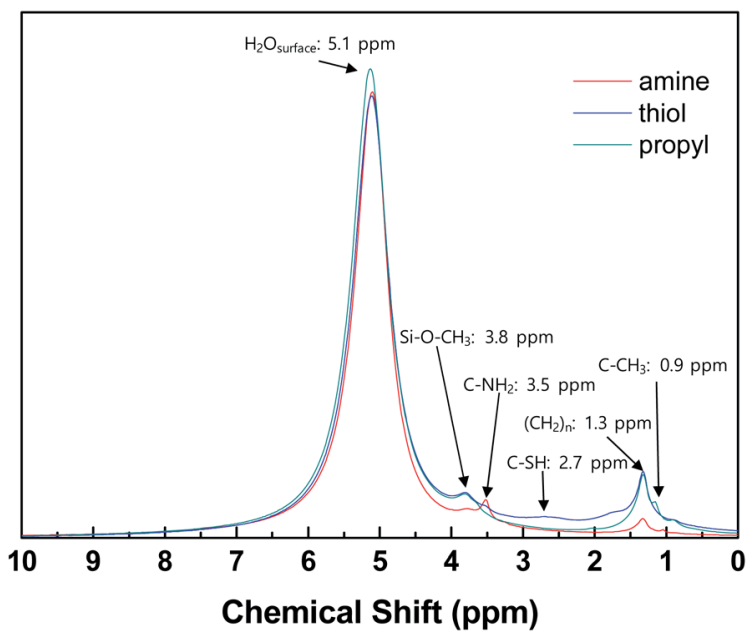

Fig. 3 1H NMR spectra of silica NPs functionalized with amine, thiol, or propyl groups.

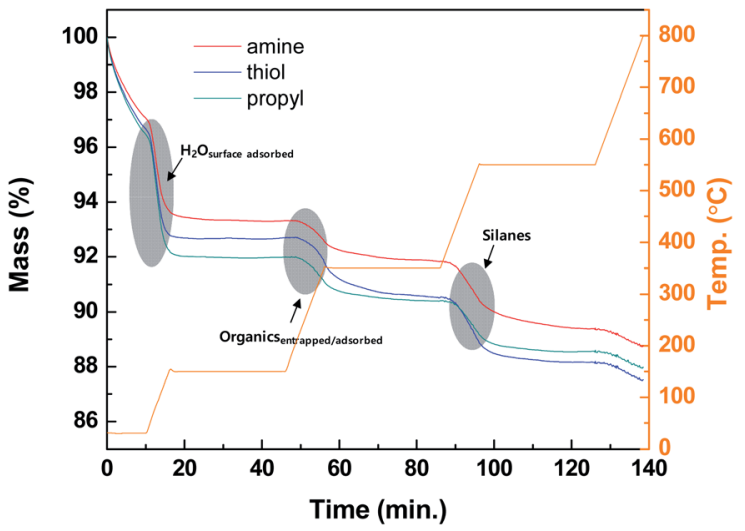

Fig. 4 TGA curves for the silica NPs functionalized with amine, thiol, or propyl groups.

the functional groups were successfully attached to the silica NP surfaces.

Table 1 summarizes the carbon $(\mathrm{C})$, nitrogen $(\mathrm{N})$, and sulfur (S) contents of the bare and modified silica NPs. None of C, N, or $\mathrm{S}$ were detected in the bare silica NPs. However, in the modified silica NPs, these elements are present in the added functional groups. The carbon contents for the amine-, thiol-, and propylmodified silica NPs are $1.27 \pm 0.06,1.59 \pm 0.02$, and $0.92 \pm$ $0.02 \%(\mathrm{w} / \mathrm{w})$, respectively. The amine-modified silica NPs contain only $0.24 \pm 0.03 \%(\mathrm{w} / \mathrm{w})$ of $\mathrm{N}$. The thiol-modified silica NPs contain only $0.85 \pm 0.06 \%(\mathrm{w} / \mathrm{w})$ of $\mathrm{S}$. These results demonstrate that the modified silica NPs contain the desired functional groups. As used equation of the surface density, the surface density of NH- $\left(\rho_{\mathrm{NH}}=6.7\right.$ molecule-S per nm $\left.{ }^{2}\right), \mathrm{SH}-\left(\rho_{\mathrm{SH}}\right.$ $=10.4$ molecule-S per $\left.\mathrm{nm}^{2}\right)$ and $\mathrm{CH}-\left(\rho_{\mathrm{CH}}=10.0\right.$ molecule-C per $\mathrm{nm}^{2}$ ) modified silica were obtained following the identical procedure. These surface densities were calculated by using the monolayer value for $\mathrm{C}_{3}$-silane grafted silica (2.1-4.2 molecule per $\left.\mathrm{nm}^{2}\right) .{ }^{53}$ Thus the functional groups are present in multilayers on the silica NP surfaces. The properties of the bare and modified silica NPs were investigated with various analytical tools and these results clearly demonstrate that the desired functional groups have been grafted onto the silica NP surfaces.

\section{Surface charges and size distributions of the silica NPs in the} decontamination foam solutions

Our SEM images show that the sizes of the silica NPs modified with various functional groups are similar and in the range 200$300 \mathrm{~nm}$ (ESI, Fig. S1†). However, the results obtained with dynamic light scattering (DLS) for the particle sizes in liquid are

Table 1 Elemental analysis results for the bare and modified silica NPs

\begin{tabular}{llll}
\hline Silica type & $\mathrm{C}(\%)$ & $\mathrm{N}(\%)$ & $\mathrm{S}(\%)$ \\
\hline Bare & 0 & 0 & 0 \\
Amine $\left(-\mathrm{NH}_{2}\right)$ & $1.27 \pm 0.06$ & $0.24 \pm 0.03$ & 0 \\
Thiol $(-\mathrm{SH})$ & $1.59 \pm 0.02$ & 0 & $0.85 \pm 0.06$ \\
Propyl $\left(-\mathrm{CH}_{3}\right)$ & $0.92 \pm 0.12$ & 0 & 0
\end{tabular}


significantly different. Before foaming, the surface charges and size distributions of the silica NPs in the decontamination solution were measured by using DLS. Considering their similar surface areas, these dramatically different dispersion behaviors are probably mainly due to the differences between the properties of the surfaces of the modified silica NPs. We determined the zeta-potentials of the silica NPs to analyze their surface charges and to evaluate the effects of $\mathrm{pH}$ on their surfaces. At $\mathrm{pH} 2.0$, the amine-modified NPs have a zeta potential of $+10.3 \pm$ $5.2 \mathrm{eV}$, which is higher than that of the bare silica NPs $(-0.1 \pm$ $4.0 \mathrm{eV})$, the thiol-modified NPs $(-2.6 \pm 4.8 \mathrm{eV})$, and the propylmodified NPs $(-2.1 \pm 3.8 \mathrm{eV})$ (Fig. 5(a)). These results show that the electrostatic repulsion between the amine-modified NPs is increased by the colloidally positive $(+)$ zeta potentials of the silica NPs, which promotes the dispersion of the silica NPs in the bulk.

Fig. 5(b) shows the sizes of the bare and amine-, thiol-, and propyl-modified silica NPs at pH 2 and $\mathrm{pH}$ 8. Particles larger than $1000 \mathrm{~nm}$ indicate the formation of big aggregates. Particle size of amine-modified silica nanoparticles at $\mathrm{pH} 2$ was $343.2 \mathrm{~nm} \pm 281 \mathrm{~nm}$. Particle sizes of stable bare, thiol-, and propyl-modified silica nanoparticles at $\mathrm{pH} 8$ were $277.9 \mathrm{~nm} \pm$ $156 \mathrm{~nm}, 370.3 \mathrm{~nm} \pm 148 \mathrm{~nm}$, and $287.4 \mathrm{~nm} \pm 167 \mathrm{~nm}$, respectively, comparable to the size of the amine-modified silica nanoparticles at $\mathrm{pH} 2$. Aggregated particle size of silica NPs depends various factors such as primary particle size, temperature, solvent permittivity, particle density, $\mathrm{pH}$, zeta potential, etc. Extended Derjaguin-Landau-Verwey-Overbeek (DLVO) theory partly can explain the stability of the particles. ${ }^{54}$ In DLVO

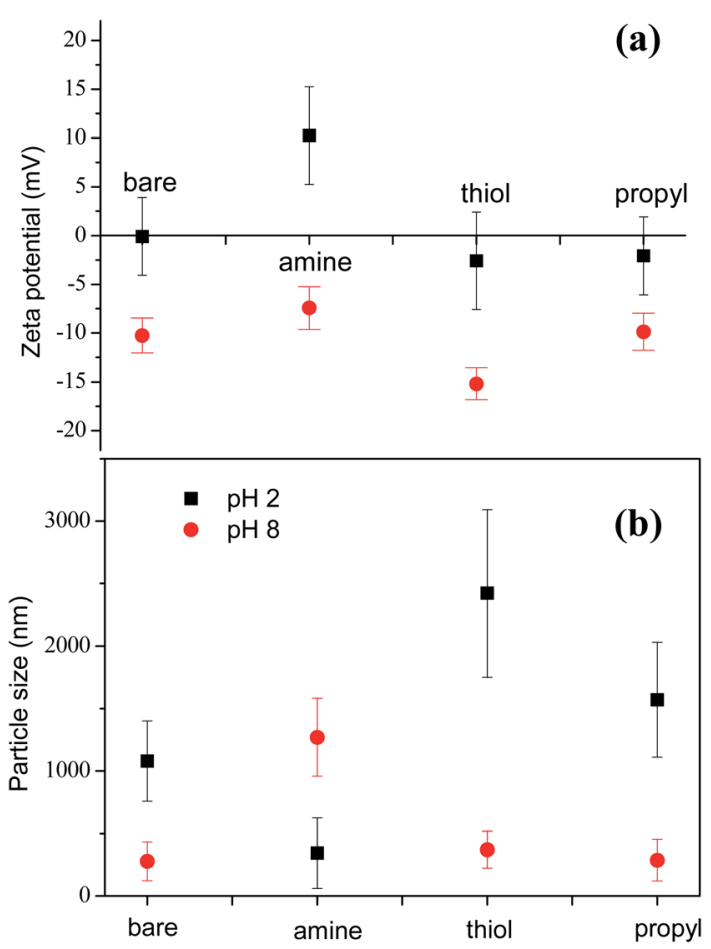

Fig. 5 (a) Zeta-potentials and (b) particle size analyses for $1 \mathrm{wt} \%$ amine-, thiol-, and propyl-modified silica NPs in $1 \%$ v/v EM 100 at pH 2 . Values are the averages of the results of three experiments. theory, total interaction potential is composed of two opposing interaction potentials: attractive van der Waals and repulsive electrostatic interaction. At $\mathrm{pH}$ 8, all particles except for the amine-modified silica particles are negatively charged, and they did not form large aggregated microparticles. Zeta potential of the amine-modified silica particles at pH $8(>-10 \mathrm{mV})$ is not enough to stabilize the particles, and it showed big aggregated particles. ${ }^{55}$ On the other hand, at $\mathrm{pH} 2$, all particles except for the amine-modified silica particles were not stable due to the small zeta potentials. In case of the positively-charged aminemodified silica at $\mathrm{pH} 2$, it did not aggregate, and the particle size is the smallest. ${ }^{56}$

\section{Turbiscan analyses of the dispersion of the silica NPs in the decontamination foam solutions}

We examined the sedimentation in the foam solutions by using an optical analyzer, the Turbiscan Tower (Formulaction, France), which characterizes the various dispersion states with
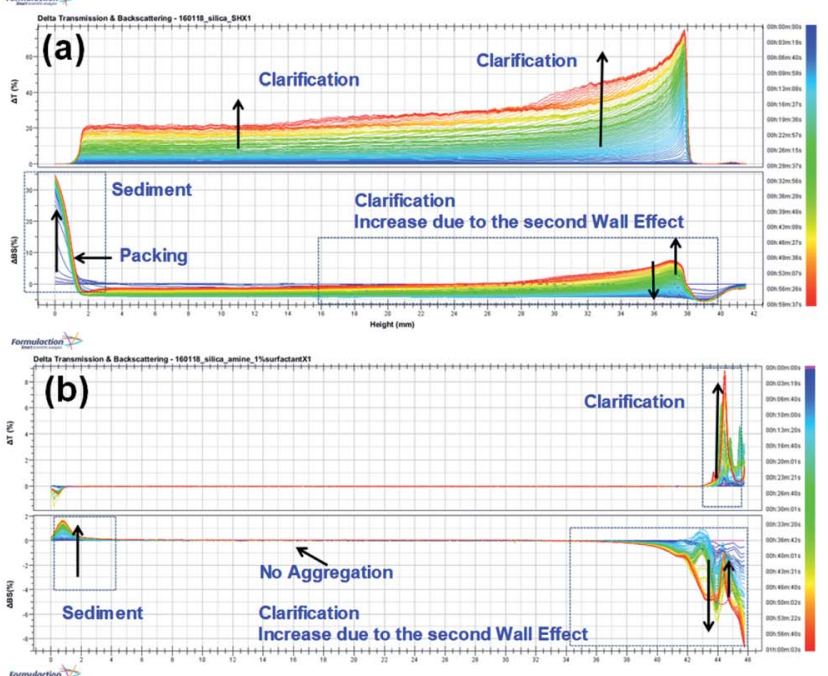

romalorition
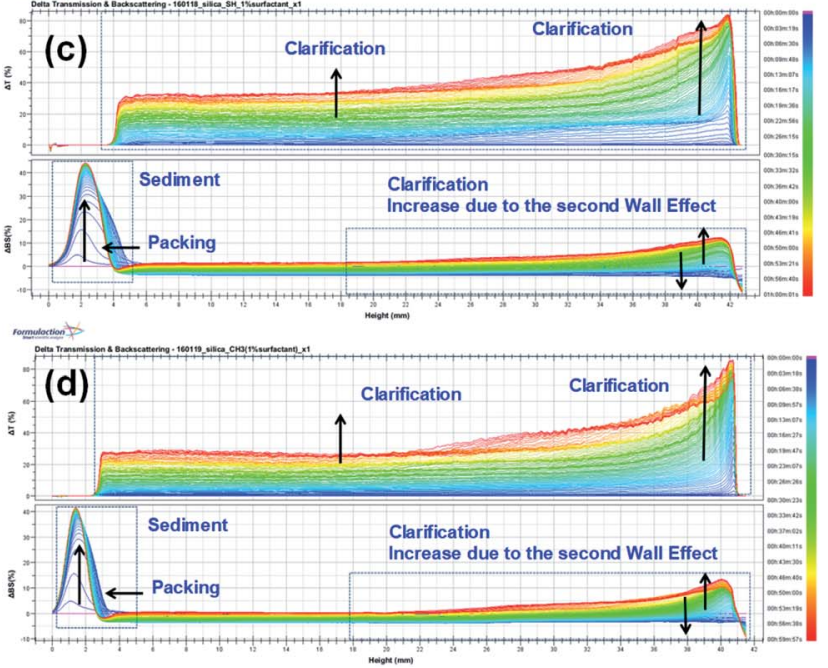

Fig. 6 Turbiscan stability kinetics and indexes for the decontamination foams containing 1 wt\% silica NPs: (a) bare, (b) amine-modified, (c) thiol-modified, and (d) propyl-modified. 


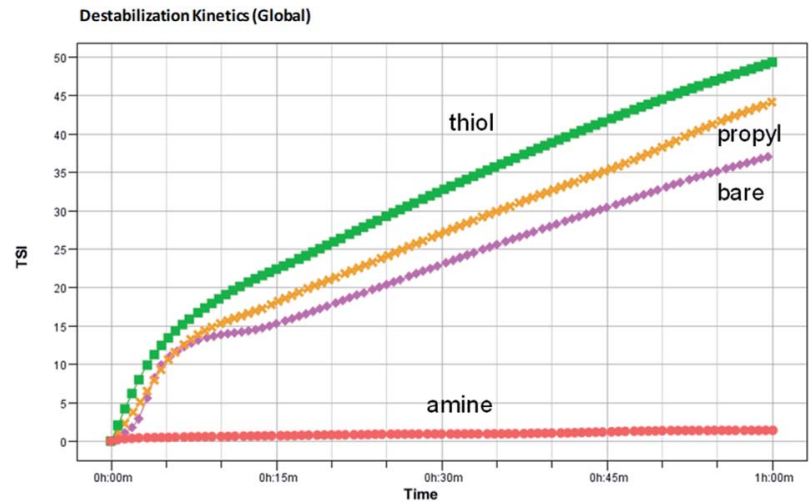

Fig. 7 Turbiscan stability kinetics and indexes for the decontamination foams containing $1 \mathrm{wt} \%$ bare, amine-, thiol-, or propyl-modified silica NPs.

multiple light scattering. The Turbiscan Tower can be used to detect and characterize destabilization processes (coalescence, flocculation, creaming and sedimentation) in dispersions with various concentrations. ${ }^{57}$ The changes in the transmission (T) and backscattering (BS) light intensity of the foams with $1 \%$ amine-, thiol-, or propyl-modified NPs are shown in Fig. 6 . The colors in the spectra correspond to different elapsed times. In Fig. 6(a)-(d), the backscattering level decreases at the top (right part of the graph) due to a reduction in the concentration of functionalized silica NPs, so clarification is occurring, whereas it increases at the bottom due to an increase in the concentration of functionalized silica NPs upon sediment formation.

The global BS decreases due to flocculation and increases due to the wall effect. The change in the global BS for aminemodified silica NPs is smaller than that for the thiol- and propyl-modified silica NPs, which indicates that the aminemodified silica NPs are more stable than the other silica NPs. BS also varies due to sedimentation. The BS at the top of the sample decreases at first but then increases due to the second wall effect after a few minutes. As a result, $\mathrm{T}$ also increases at the same sample height. The BS in the middle steadily increases due to the second wall effect, but flocculation was not evident during the analysis. When we ranked the stability of the NPs using TSI (global), the amine-modified silica NPs were found to be more stable than the thiol- and propyl-modified silica NPs. Using the TSI values shown in Fig. 7, we can list the foams in the order of decreasing stability as follows: amine-modified $>$ bare $>$ propyl-modified $>$ thiol-modified. The TSI values of the aminemodified silica NPs in sedimentation are smaller than those of the other silica NPs, which suggests that the sedimentation of the amine-modified silica NPs is slower, so they are better dispersed in complex fluids than the other silica NPs.

\section{Stabilities of the decontamination foams containing modified silica NPs}

The variations with time in the total height of the foams are shown in Fig. 8. The total height of the foam containing aminemodified silica NPs is stable for a relatively long period, whereas the heights of the foams containing bare, thiol-, or propylmodified silica NPs decrease to $50 \%$ after $60 \mathrm{~min}$, i.e. their

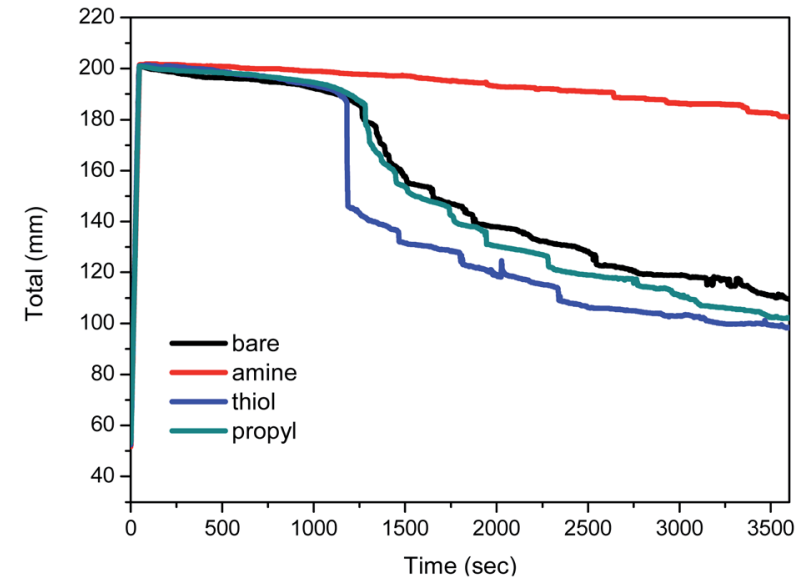

Fig. 8 Stability results for foams containing $1 \mathrm{wt} \%$ bare, amine-, thiol-, and propyl-modified silica NPs and $1 \% \mathrm{v} / \mathrm{v}$ non-ionic surfactant with a $\mathrm{pH}$ of 2 . Values are the averages of the results of three experiments.

foam volumes decreased to $100 \mathrm{~mL}$. In particular, the foam containing $1 \mathrm{wt} \%$ amine-modified silica NPs at $\mathrm{pH} 2$ retains its initial height for $60 \mathrm{~min}$. A previous study reported that the presence of a non-ionic surfactant does not affect foam stability at $\mathrm{pH} 2,3$, or 5 , so it can be used under acidic $\mathrm{pH}$ conditions. ${ }^{12}$ Ethoxylated fatty alcohols are important non-ionic surfactants and are thought to be hydrolytically stable in the $\mathrm{pH}$ range 3$11 .{ }^{58}$ However, low $\mathrm{pH}$ values $(\mathrm{pH}<3)$ affect the foam stability when diluted nitric acid is used as the chemical reagent. After the addition of silica NPs, the foam was stable for $1 \mathrm{~h}$. The silica NPs form a dense aggregation in the liquid film at the foam surface; this phenomenon is an important factor that increases the foam stability while preventing foam coalescence.

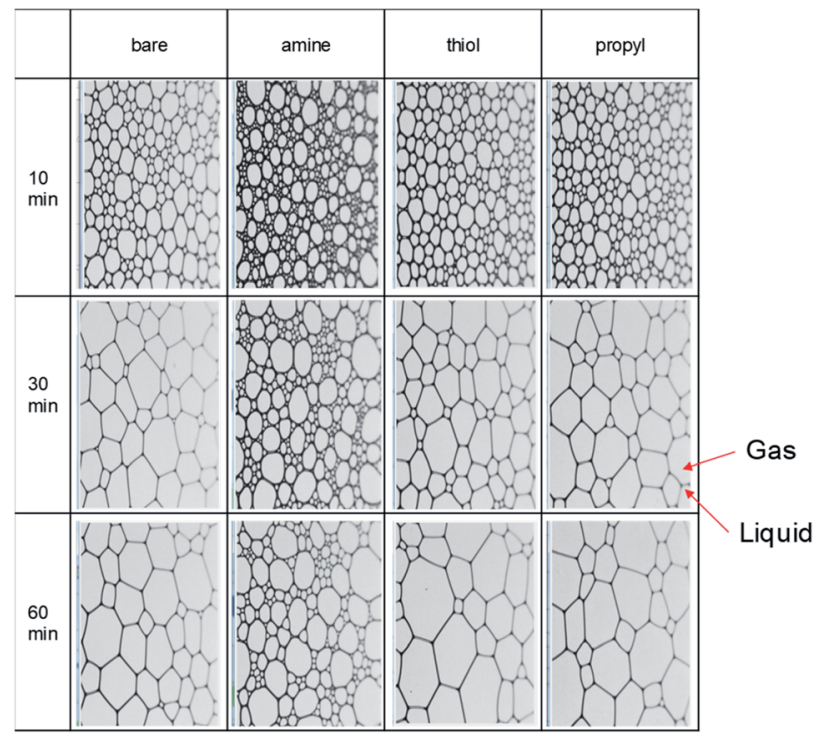

Fig. 9 Images of the bubbles and structures in 1\% v/v EM $100(\mathrm{pH} 2)$ containing 1 wt\% amine-, thiol-, and propyl-modified silica NPs after 10,30 , and $60 \mathrm{~min}$. 

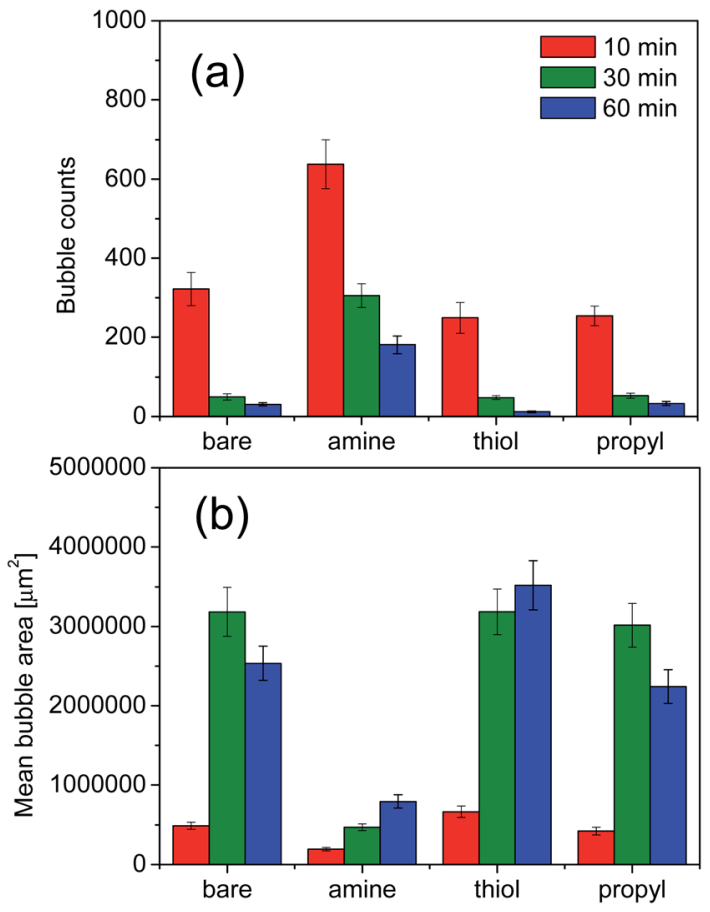

Fig. 10 Results of the foam structure measurements at $\mathrm{pH} 2$ : (a) bubble counts and (b) bubble areas. Values are the averages of the results of three experiments.

\section{Structures of the decontamination foams containing modified silica NPs}

We evaluated the stabilities of the decontamination foams containing surface-modified silica NPs; Fig. 9 and 10 show the variations in the structures and stabilities of the decontamination foams prepared using $1 \mathrm{wt} \% \mathrm{~mol}_{\text {silane }} \mathrm{mol}_{\text {silica }}{ }^{-1}$ bare silica NPs and silica NPs modified with amine, thiol, and propyl groups at pH 2 over a 60 min period. Fig. 9 shows the structures of the decontamination foams at times of 10,30, and $60 \mathrm{~min}$ after foaming. Fig. 10 shows the bubble counts and mean areas of the decontamination foams in Fig. 9. Since the efficiency of the removal of contaminants obtained with a decontamination foam is very important, we performed foam stability tests for the decontamination foams prepared in this study under acidic conditions at $\mathrm{pH} 2$. The bubble count for the foam with aminemodified silica NPs, i.e., 200, is approx. 4.4-10 times larger than those obtained for the foams with bare silica NPs (40), thiolmodified silica NPs (20), and propyl-modified silica NPs (45) after 60 min (Fig. 10(a)). This result confirms that the stability of the decontamination foam containing amine-modified silica NPs is better than those of the other formulations we tested. The mean bubble size of the foam with amine-modified silica NPs, i.e., $795088 \mu \mathrm{m}^{2}$, after $60 \mathrm{~min}$ is approx. 2.5-4 times smaller than those of the foams with bare silica NPs (2 536079 $\mu \mathrm{m}^{2}$ ), thiol-modified silica NPs (3 $519523 \mu \mathrm{m}^{2}$ ), and propylmodified silica NPs (2 $243129 \mu \mathrm{m}^{2}$ ) (Fig. 10(b)). These results show that the bubble size of the decontamination foam containing amine-modified silica NPs is less than those of the foams containing NPs modified with other functional groups, and that this foam has a higher bubble count.

The structural properties of the bubbles, namely their sizes, numbers and average areas, were determined with a DFA 100. Previous studies have reported that in the absence of silica NPs the bubbles grow and became irregular over time, and that larger bubbles merge with adjacent small bubbles due to pressure differences according to the Young-Laplace theory. In contrast, the presence of partially hydrophobic or surfactantsilica NPs keeps the bubbles small; the added NPs are adsorbed onto the foam surface and improve the deformation resistance of the foam film, thus increasing foam stability. ${ }^{59}$ In addition, the small amount of floc resulting from combining the surfactant with the NPs improves the foam stability by retarding foam coalescence. ${ }^{60}$ The resultant large number of small bubbles with uniform size results in comparatively good foamability and foam stability. ${ }^{44,61}$ For foams containing amine-modified silica NPs, the average count and mean area of bubbles are the highest and lowest respectively of the foams prepared in this study for all analyzed durations. This result indicates that amine-modified silica NPs provide more stability than thiol- or propyl-modified silica NPs and bare silica NPs.

\section{Mechanism of the stabilization of foams by surface-modified silica NPs}

Various mechanisms have been proposed to explain the enhancement of foam stability by NPs. The clusters of NPs form a network inside the liquid film in the foam, which enhances foam stability by preventing coalescence and coarsening. ${ }^{10}$

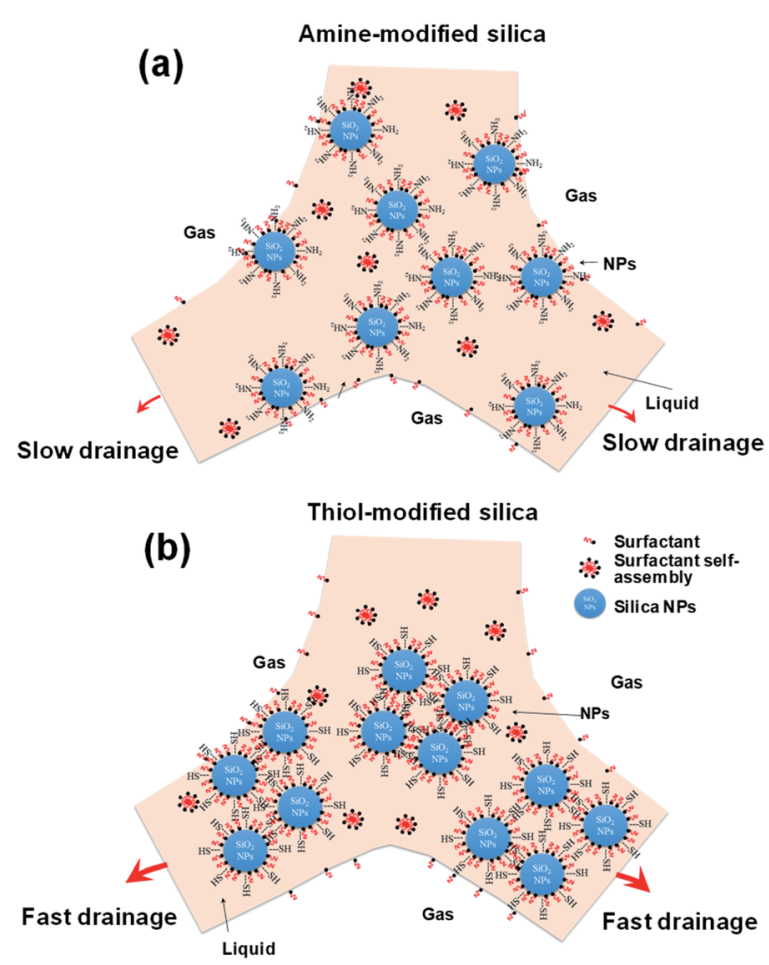

Fig. 11 Schematic diagram of the mechanism of foam stability for two different surface-modified silica NPs. 
Dickson proposed that the dominant mechanism of foam stabilization by NPs is the aggregation of NPs at the solutiongas interface, which acts as a barrier preventing film breakage. ${ }^{62}$ Another proposal suggests that the improvement in foam stability in the presence of NPs is due to the decrease in interfacial tension. ${ }^{23}$ Some studies concluded that the partial flocculation of NPs with appropriate size in the bulk and at the interface is the key mechanism in the improvement of foam stability. ${ }^{\mathbf{1 0 6 3 , 6 4}}$ AIYousef reported that the addition of more solid particles or surfactant has a negative impact on foam stability and that an intermediate concentration of NPs with respect to that of the surfactant is required to enhance foam stability. ${ }^{\mathbf{6 0}}$

Fig. 11 shows schematic diagrams of the behaviors of highly dispersed silica NPs (amine-modified) and agglomerated silica NPs (thiol- and propyl-modified) in the liquid films of decontamination foams. The results in Fig. 5-7 show that the agglomeration of the amine-modified silica NPs is less than that of the other modified silica NPs due to their thorough dispersion in the liquid film. Thus, well-dispersed and smaller silica NPs persist for a longer time in the liquid film than larger silica NPs, which prevents drainage and coalescence, and thus enhances foam stability. In addition, the viscosity of amine silica NPs increased, because more smaller NPs are present per unit volume (ESI, Fig. S2 $\dagger$ ). AIYousef showed that the generation of flocs as a result of mixing NPs and surfactant can enhance the foam stability by providing a barrier between the gas bubbles and thus delaying their coalescence. In addition, NPs increase the solution viscosity of thin aqueous films and thereby slow their drainage rate. ${ }^{60}$ As a result, an intermediate concentration of NPs with respect to that of the surfactant enhances the foam stability.

However, the agglomeration of the thiol- and propylmodified silica NPs occurs rapidly due to their neutral and partially hydrophobic surfaces. Agglomerated particles (1000$2500 \mathrm{~nm}$ ) undergo rapid drainage and do not remain in the liquid film. AIYousef reported that the aggregation of NPs at these concentrations results in the formation of flocs with large diameters and a reduction in the maximum capillary pressure of coalescence, and so expedites film breakage. ${ }^{60}$ Therefore, even though the viscosity might increase because of agglomeration, the maximum capillary pressure of coalescence will decrease as the radius of the particles increases.

\section{Conclusions}

This study assessed the effects of the addition of modified silica NPs to decontamination foams on their stability with the aim of decreasing the amounts of waste generated by chemical decontamination and increasing the decontamination efficiency. We concluded that the use of amine-modified silica NPs with a non-ionic surfactant in decontamination foams with a pH of 2 provides better foam stability than the addition of bare, propyl-, and thiol-modified silica NPs. The stability of a decontamination foam containing amine-modified silica NPs is approximately 5-8 times higher than foams containing silica NPs modified with other functional groups because of its thorough dispersion and higher viscosity. The addition of amine-modified silica NPs results in a larger number of particles per unit volume and in smaller particles than the other modified silica NPs because they have a partial positive charge, which prevents aggregation between particles and increases their dispersibility in the liquid film of the decontamination foam. Such a decontamination foam with surface-modified silica NPs can be applied to radionuclide-contaminated areas as a highly effective decontamination agent for the reduction of secondary waste.

\section{Conflicts of interest}

There are no conflicts to declare.

\section{Acknowledgements}

This study was supported by National Research Foundation of Korea (NRF) grants funded by the Korea government (MSIT) (No. 2020M2A8A5079107 \& No. 2017M2A8A5015148). The authors thank Jong Hwan Baek (Leanontech Co., Korea) and Jong-Yun Kim (KAERI) for their helps and valuable suggestions regarding the Turbiscan data analysis and particle size distributions.

\section{References}

1 C. Dame, C. Fritz, O. Pitois and S. Faure, Curr. Opin. Colloid Interface Sci., 2005, 263, 210-218.

2 A. Barbetta, G. Rizzitelli, R. Bedini, R. Pecci and M. Dentini, Soft Matter, 2010, 6, 1785-1792.

3 C. Colosi, M. Costantini, A. Barbetta, R. Pecci, R. Bedini and M. Dentini, Langmuir, 2013, 29, 82-91.

4 R. Deleurence, T. Saison, F. Lequeux and C. Monteux, Soft Matter, 2015, 11, 7032-7037.

5 T. Andersen, J. E. Melvik, O. Gåserød, E. Alsberg and B. E. Christensen, Biomacromolecules, 2012, 13, 3703-3710.

6 T. Andersen, J. E. Melvik, O. Gåserød, E. Alsberg and B. E. Christensen, Carbohydr. Polym., 2014, 99, 249-256.

7 I. Lesov, S. Tcholakova and N. Denkov, J. Colloid Interface Sci., 2014, 426, 9-21.

8 Q. Liu, S. Zhang, D. Sun and J. Xu, Colloids Surf., A, 2009, 338, 40-46.

9 Q. Liu, S. Zhang, D. Sun and J. Xu, Colloids Surf., A, 2010, 355, 151-157.

10 S. Zhang, Q. Lan, Q. Liu, J. Xu and D. Sun, Colloids Surf., A, 2008, 317, 406-413.

11 S. Guignot and S. Faure, Decontamination, Stripping And/or Degreasing Foam Containing Solid Particles, 2010.

12 I. H. Yoon, C. H. Jung, S. B. Yoon, S. Y. Park, J. K. Moon and W. K. Choi, Ann. Nucl. Energy, 2014, 73, 168-174.

13 H. Rezvani, D. Panahpoori, M. Riazi, R. Parsaei, M. Tabaei and F. B. Cortés, J. Mol. Liq., 2020, 304, 112730.

14 H. Zhou, C. Dai, Q. Zhang, Y. Li, W. Lv, R. Cheng, Y. Wu and M. Zhao, J. Mol. Liq., 2019, 293, 111500.

15 Y. Chevalier and M. A. Bolzinger, Colloids Surf., A, 2013, 439, 23-34. 
16 P. Degen, D. C. F. Wieland, S. Leick, M. Paulus, H. Rehage and M. Tolan, Soft Matter, 2011, 7, 7655-7662.

17 A. Maestro, E. Guzmán, F. Ortega and R. G. Rubio, Curr. Opin. Colloid Interface Sci., 2014, 19, 355-367.

18 D. Zabiegaj, E. Santini, E. Guzmán, M. Ferrari, L. Liggieri, V. Buscaglia, M. T. Buscaglia, G. Battilana and F. Ravera, Colloids Surf., A, 2013, 438, 132-140.

19 T. N. Hunter, R. J. Pugh, G. V. Franks and G. J. Jameson, Adv. Colloid Interface Sci., 2008, 137, 57-81.

20 Z. G. Cui, K. Z. Shi, Y. Z. Cui and B. P. Binks, Colloids Surf., A, 2008, 329, 67-74.

21 Y. Zhu, J. Jiang, K. Liu, Z. Cui and B. P. Binks, Langmuir, 2015, 31, 3301-3307.

22 B. P. Binks, J. A. Rodrigues and W. J. Frith, Langmuir, 2007, 23, 3626-3636.

23 Q. Lan, F. Yang, S. Zhang, S. Liu, J. Xu and D. Sun, Colloids Surf., A, 2007, 302, 126-135.

24 L. Liggieri, E. Santini, E. Guzmán, A. Maestro and F. Ravera, Soft Matter, 2011, 7, 7699-7709.

25 A. Maestro, E. Rio, W. Drenckhan, D. Langevin and A. Salonen, Soft Matter, 2014, 10, 6975-6983.

26 B. A. Noskov, P. A. Yazhgur, L. Liggieri, S. Y. Lin, G. Loglio, R. Miller and F. Ravera, Colloid J., 2014, 76, 127-138.

27 F. Ravera, E. Santini, G. Loglio, M. Ferrari and L. Liggieri, J. Phys. Chem. B, 2006, 110, 19543-19551.

28 F. Ravera, M. Ferrari, L. Liggieri, G. Loglio, E. Santini and A. Zanobini, Colloids Surf., A, 2008, 323, 99-108.

29 P. Esmaeilzadeh, N. Hosseinpour, A. Bahramian, Z. Fakhroueian and S. Arya, Fluid Phase Equilib., 2014, 361, 289-295.

30 H. Ma, M. Luo and L. L. Dai, Phys. Chem. Chem. Phys., 2008, 10, 2207-2213.

31 M. Zargartalebi, N. Barati and R. Kharrat, J. Pet. Sci. Eng., 2014, 119, 36-43.

32 H. Vatanparast, A. Samiee, A. Bahramian and A. Javadi, Colloids Surf., A, 2017, 513, 430-441.

33 M. Zargartalebi, R. Kharrat and N. Barati, Fuel, 2015, 143, 21-27.

34 A. Maestro, E. Guzmán, E. Santini, F. Ravera, L. Liggieri, F. Ortega and R. G. Rubio, Soft Matter, 2012, 8, 837-843.

35 L. R. Arriaga, W. Drenckhan, A. Salonen, J. A. Rodrigues, R. Íñiguez-Palomares, E. Rio and D. Langevin, Soft Matter, 2012, 8, 11085-11097.

36 R. Deleurence, C. Freymond, T. Saison, F. Lequeux and C. Monteux, Colloids Surf., A, 2017, 534, 2-7.

37 H. Kang, D. J. Long and C. L. Haynes, Langmuir, 2019, 35, 7985-7994.

38 C. H. Lee, S. H. Park, W. Chung, J. Y. Kim and S. H. Kim, Colloids Surf., A, 2011, 384, 318-322.

39 H. Vatanparast, F. Shahabi, A. Bahramian, A. Javadi and R. Miller, Sci. Rep., 2018, 8, 7251.

40 Q. Zhu, H. L. Zhou, Y. X. Song, Z. D. Chang and W. J. Li, Int. J. Miner., Metall. Mater., 2017, 24, 208-215.
41 K. Monjezi, M. Mohammadi and A. R. Khaz'ali, J. Mol. Liq., 2020, 311, 113043.

42 S. B. Yoon, I. H. Yoon, C. Kim, C. H. Jung, S. Kim, W. K. Choi and J. K. Moon, Asian J. Chem., 2014, 26, 1401-1404.

43 C. Bilke-Krause, T. Schörck and T. Winkler, Studies on the Stability of Foams: KRÜSS Application No AN267e, 2010, pp. 1-6.

44 K. Oetjen, C. Bilke-Krause, M. Madani and T. Willers, Colloids Surf., A, 2014, 460, 280-285.

45 M. C. Brochier Salon, P. A. Bayle, M. Abdelmouleh, S. Boufi and M. N. Belgacem, Colloids Surf., A, 2008, 312, 83-91.

46 B. Grünberg, T. Emmler, E. Gedat, I. Shenderovich, G. H. Findenegg, H. H. Limbach and G. Buntkowsky, Chem.-Eur. J., 2004, 10, 5689-5696.

47 J. Zou and S. M. Kauzlarich, J. Cluster Sci., 2008, 19, 341-355. 48 W. H. Chen, Y. T. Tseng, S. Hsieh, W. C. Liu, C. W. Hsieh, C. W. Wu, C. H. Huang, H. Y. Lin, C. W. Chen, P. Y. Lin and L. K. Chau, RSC Adv., 2014, 4, 46527-46535.

49 T. Gunji, Y. Shigematsu, T. Kajiwara and Y. Abe, Polym. J., 2010, 42, 684-688.

50 L. Ma, S. Dong, Z. He, Y. Yang, H. Jia, Y. Di, Y. Tang and Y. Wang, RSC Adv., 2016, 6, 98761-98767.

51 A. Şengül and H. Arslan, Turk. J. Chem., 2008, 32, 355-364.

52 V. Dugas and Y. Chevalier, Langmuir, 2011, 27, 14188-14200.

53 R. G. Acres, A. V. Ellis, J. Alvino, C. E. Lenahan, D. A. Khodakov, G. F. Metha and G. G. Andersson, J. Phys. Chem. C, 2012, 116, 6289-6297.

54 P. P. Atkins and J. de, Atkin's Physical Chemistry, Oxford University Press, 8th edn, 2006.

55 J. D. Clogston and A. K. Patri, in Characterization of Nanoparticles Intended for Drug Delivery, ed. S. E. McNeil, Humana Press, Totowa, NJ, 2011, pp. 63-70, DOI: 10.1007/ 978-1-60327-198-1_6.

56 R. P. Bagwe, L. R. Hilliard and W. Tan, Langmuir, 2006, 22, 4357-4362.

57 S. Perumal, A. Raji and I. W. Cheong, Langmuir, 2018, 34, 6737-6747.

58 K. Holmberg, B. Jönsson, B. Kronberg and B. Lindman, Surfactants and Polymers in Aqueous Solutions, 2003.

59 Q. Lv, Z. Li, B. Li, S. Li and Q. Sun, Ind. Eng. Chem. Res., 2015, 54, 9456-9477.

60 Z. A. AlYousef, M. A. Almobarky and D. S. Schechter, J. Colloid Interface Sci., 2018, 511, 365-373.

61 J. Chen, M. Qiao, N. Gao, Q. Ran, S. Wu and S. Qi, Colloids Surf., A, 2017, 522, 593-600.

62 E. Dickinson, Curr. Opin. Colloid Interface Sci., 2010, 15, 4049.

63 B. P. Binks and J. A. Rodrigues, Langmuir, 2007, 23, 74367439.

64 C. P. Whitby, D. Fornasiero and J. Ralston, J. Colloid Interface Sci., 2008, 323, 410-419. 